\title{
Modelling the Dynamical Behaviour of Nuclear Reactor Fuel Assemblies
}

\author{
J. Planchard \\ Electricité de France, Direction des Etudes et Recherches, 1 av. du Général de Gaulle, \\ F-92141 Clamarl, France
}

Abstract

The computation of the motion of fuel rods of nuclear reactor cores is generally difficult for the number of elements is large. This paper is devoted with an homogenization process likening the fluid-tube-spacer system as an equivalent elastic medium for which the constitutive laws are derived. Numerical schemes are briefly described.

\section{Introduction}

The mechanical behaviour of the fuel clads of nuclear reactors is of great importance with regards to safety. Indeed, the fuel elements can be subjected to flow-induced vibrations, seismic impact, pressure drop due to LOCA accident, etc.. ; it is then necessary to know a priori the displacement of the entire core, taking into account presence of the cooling fluid. Unfortunately, direct numerical calculations are not possible to carry out, because of the large number of fuel rods, about 45,000 for PWR cores. The sole remedy consists by replacing the fluid-fuel assembly coupled system by an equivalent homogeneous material. This method is general and is known as homogenization in the 1iterature (see [1], $[2]$ ).

In the following section, we outline this theory in case of fluid-tube bundle interaction.

\section{Basic equations}

In order to simplify the presentation, we consider two-dimension problens, and we suppose that the fluid is perfect and incompressible. The rods are rigid, elastically mounted, linked by springs of stiffness $k$. In this situation, the motion equation of each rod is (see figures 1 and 2) :

$$
m \frac{\overrightarrow{d^{2} s_{0}}}{d t^{2}}=\int_{Y_{0}} p(x, t) \vec{n}_{x} d Y_{0}-k \sum_{l=1}^{4}\left(\vec{s}_{\ell}(t)-\vec{s}_{0}(t)\right)-k_{0} \vec{s}_{0}(t) .
$$

where $p$ is the pressure, $\vec{s}_{0}$ and $\vec{s}_{\ell}$ are respectively the displacements of the tube $Y_{0}$ and its neighbouring ones ; $k_{0}$ is the stiffness of each cylinder; $\overrightarrow{\mathrm{n}}_{\mathrm{x}}$ is the normal unit on $\gamma_{\mathrm{o}}$, oriented inside $\gamma_{0}\left(\gamma_{0}\right.$ denotes also the wall tube). The rod mass m, $\mathrm{k}$ and $\mathrm{k}_{\mathrm{o}}$ are considered per length unit. $p$ is related to the speed potential of fluid $\phi$ by

$$
p=-\rho_{0} \frac{\partial \phi}{\partial t}, \quad \rho_{0}: \text { fluid specific density. }
$$


Incompressibility implies :

$\Delta \phi=0$ in $\Omega_{0}$, domain occupied by the fluid.

The boundary conditions satisfied by $\phi$ are :

$\frac{\partial \phi}{\partial \mathrm{n}}(\mathrm{x})=\frac{\overrightarrow{\mathrm{ds}} \overrightarrow{\mathrm{o}}_{\mathrm{d}}}{\mathrm{dt}} \cdot \overrightarrow{\mathrm{n}}_{\mathrm{x}}$ on each $\gamma_{\mathrm{o}}, \frac{\partial \phi}{\partial \mathrm{n}}=0$ on $\Gamma$,

$\Gamma$ being the rigid wall of the cavity containing the bundle.

Introducing the added mass matrix $\mathrm{pH}_{0}$ calculated by the influence functions (Chen [3], Paidoussis [4], see also [5]), and the stiffness matrix $S$ of the spring-tube system, we obtain, eliminating $\varnothing$ :

$$
\left(m+\rho_{o} H\right) \frac{\overrightarrow{d^{2} s}}{d t^{2}}+\vec{s} \vec{s}=0,
$$

where $\vec{s}$ is the vector of order $2 \mathrm{~N}$ made up with the displacement-vectors $\vec{s}_{0}$, $\mathrm{N}$ being the total number of tubes.

\section{Hornogenization of the equations}

For this operation, it is necessary to assume that the rods are identical (same $\mathrm{m}, \mathrm{k}$, $k_{0}$ and same radius) and the bundle is spatially periodic with a tube pitch $E$ small compared to the dimensions of the core. Multiplying eq. (3) by an arbitrary regular rest-function $\mathrm{v}(\mathrm{x})$, integrating by parts on $\Omega_{0}$ and using the boundary conditions (4), we obtain :

$$
\int_{\Omega_{0}} \operatorname{grad} \phi(x, t) \cdot \operatorname{grad} v(x) d x=\sum_{\ell=1}^{K} \frac{\overrightarrow{d s} \ell}{d t} \cdot \overrightarrow{\mathrm{N}_{\ell} \vec{v}}
$$

where $\overrightarrow{\mathrm{N}_{\ell}} \overrightarrow{v^{\mathrm{V}}}=\int_{\gamma_{\ell}} \mathrm{v}(\mathrm{x}) \overrightarrow{\mathrm{n}}_{\mathrm{x}} \mathrm{d} \gamma_{\ell}$, $\ell$ refers to the $\ell^{\text {th }}$ tube in the entire bundle.

Because $\varepsilon$ is small, an asymptotic analysis (see [6], [7]) leads to replace the first term of eq. (5) by $B \int_{\Omega} \operatorname{grad} \phi \cdot \operatorname{grad} v \mathrm{dx}$.

Here $\Omega$ denotes the region occupied by both the fluid and the bundle. $\beta$ is calculated as follows : let us introduce the functions $X_{i}(x)$ on the elementary cell $C$ (see figure 3 ) satisfying :

$$
\left\{\begin{array}{l}
\Delta x_{i}(x)=0 \text { in } C^{*}, \frac{\partial x_{i}}{\partial n}(x)=-\cos \left(\vec{n}, x_{i}\right) \text { on } \gamma, \\
x_{i}\left(x_{1}+\varepsilon, x_{2}\right)=x_{i}\left(x_{1}, x_{2}+\varepsilon\right)=x_{i}\left(x_{1}, x_{2}\right), \quad \forall x .
\end{array}\right.
$$

Then we define

$$
\left.\beta_{i j}=\frac{1}{\varepsilon^{2}-\pi r^{2}} \int_{C^{*}}\left(\frac{\partial x_{i}}{\partial x_{j}}(x)+\delta_{i j}\right) d x, i, j=1 \text { and } 2 \text { (then } \beta_{i j}=\beta_{j i}\right) \text {. }
$$

Geometric symmetries imply that $\beta_{12}=0$ and $\beta_{11}=\beta_{22}=\beta$ (say).

On the other hand, it can be shown that :

$\overrightarrow{\mathrm{N}_{\ell} \mathrm{v}}=-\pi r^{2} \operatorname{grad} \mathrm{v}\left(\mathrm{x}_{\ell}\right)+o\left(\varepsilon^{3}\right)$,

where $x_{\ell}$ is the center of the circle $\gamma_{\ell}$, so that we can make the approximation :

$\sum_{\ell=1}^{K} \frac{\overrightarrow{d s} \ell}{d t} \cdot \overrightarrow{N_{\ell}} \simeq-\frac{\pi r^{2}}{\varepsilon^{2}} \int_{\Omega} \frac{\partial \vec{s}}{\partial t} \cdot \operatorname{grad} v(x) d x=\frac{\pi x^{2}}{\epsilon^{2}} \frac{d}{d t} \int_{\Omega} \operatorname{div} \vec{s}^{2}(x, t) v(x) d x$. 
In this transformation, we have replaced the $\overrightarrow{s_{\ell}}$ by a continuous field $\vec{s}(x, t)$; it is also assumed, in a aim of simplification, that the tubes are fixed near the boundary $\Gamma$ (see [6] for more realistic boundary conditions). Because $v$ is arbitrary, relation (6) 1eads to

$-\beta \Delta \phi(x, t)=\frac{\pi r^{2}}{\varepsilon^{2}} \operatorname{div} \frac{\partial \vec{s}}{\partial t}(x, t)$.

Now, it remains to homogenize the rod motion equation. It is noted that $\sum_{\ell=1}^{4}\left(\vec{s}_{0}-\vec{s}_{\ell}\right)$ is a five-points approximation by finite difference of $-\varepsilon^{2} \overrightarrow{\Delta s}(x, t)$, so that eq. (1) becomes, after some handling :

$$
\left(-k E^{2} \Delta+k_{0}+m \frac{\partial^{2}}{\partial t^{2}}\right) \stackrel{r}{s}(x, t)=\pi r^{2} \rho_{0} \operatorname{grad} \frac{\partial \phi}{\partial t}(x, t) .
$$

The boundary conditions associated to (7), (8) are :

$$
\overrightarrow{\mathrm{s}}=0, \frac{\partial \phi}{\partial \mathrm{n}}=0 \text { on } \mathrm{r} \text {. }
$$

Numerical scheme : a semi-explicit scheme can be used, for instance :

$$
\left\{\begin{array}{l}
\left(-k \varepsilon^{2} \Delta+k_{0}\right) \vec{s}^{n}+m \frac{\vec{s}^{n+1}-2 s^{n}+s^{n-1}}{\Delta t^{2}}=\pi r^{2} \rho_{0} \operatorname{grad} \frac{\phi^{n}-\phi^{n-1}}{\Delta t}, \\
-\beta \Delta \frac{\phi^{n+1}+\phi^{n}}{2}=\frac{\pi r^{2}}{E^{2}} \operatorname{div} \frac{\vec{s}^{n+1}-\vec{s}^{n}}{\Delta t},
\end{array}\right.
$$

where the index n means that the quantities must be considered at time $n \Delta t$. Of course, a stability condition holds. Fully implicit schemes can be also defined. The different differential operators with respect to $x$ are approached by standard finite element method.

\section{Remarks :}

1 -Absence of mechanical links between adjacent assemblies can be considered : equations

(7), (8) are only valid inside each assembly and it is necessary to add equations at each common interface (see [6]).

2 - Replacing $\partial / \partial t$ by $i \omega$, it can be shown that equations (6), (7) have an infinite discrete set of real eigenfrequencies $\omega_{n}$ which tend to infinity if $k>0$ (presence of spacers) while these eigenvalues lie inside a finite interval when $k=0$ (absence of spacer).

\section{Extension to three-dimension case}

Because the fuel rods are long, we have to take into account their bending, and moreover the presence of an axial flow with velocity $n$. $\phi$ is concerned only with the motion of fluid generated by the transverse displacement of the tubes, so that the homogenized equations are (see [6]), z being the axial coordinate :

$$
\left\{\begin{array}{l}
\left(m \frac{\partial^{2}}{\partial t^{2}}-k \varepsilon^{2} \Delta_{x}+E I \frac{\partial^{4}}{\partial z^{4}}\right) \vec{s}(x, z, t)=\pi r^{2} \rho_{0} \operatorname{grad}_{x}\left(\frac{\partial}{\partial t}+U \frac{\partial}{\partial z}\right) \phi(x, z, t), \\
-\beta \Delta_{x} \phi(x, z, t)=\frac{\pi r^{2}}{\varepsilon^{2}} \operatorname{div} \frac{\partial \vec{s}}{\partial t}(x, z, t) .
\end{array}\right.
$$

Here, $\Delta_{x}=\frac{\partial^{2}}{\partial x_{1}{ }^{2}}+\frac{\partial^{2}}{\partial x_{2}{ }^{2}}, \operatorname{grad}_{x}=\operatorname{col}\left(\frac{\partial}{\partial x_{1}}, \frac{\partial}{\partial x_{2}}, 0\right), \vec{s}-\operatorname{col}\left(s_{1}, s_{2}, 0\right) ;$ EI is the rod stiffness. The term in $v$ results from the Lightfil1's theory (see [_8], [_9] for instance). 
of course, we must add two boundary conditions at each tube end. Numerical schemes of the type (10) can be used for solving equation (11).

$\underline{\text { Remarks : }}$

1 - The method is easily extended to fast breeder reactor cores (figures 4 and 5 ). It can be seen that the homogenized constitutive laws of fuel pins are similar to eq. (11), except that $\beta \Delta_{x}$ must be replaced by div ( $\beta$ grad.), where $\beta$ is a matrix. In the same way, it is possible to obtain the equations governing the global displacements of the assemblies considered as beams, in which case the term $\Delta x_{x} \vec{s}$ disappears because there is no spacer between the adjacent ones : a computer code based upon this method is presently in course of creation at C.E.A. (Centre d'Etudes Nucléaires, Saclay).

2 - Another homogenization process based on averaging the conservation laws, has been investigated by Benner and Schumann $[10]$.

\section{Conclusion}

We have transformed, via homogenization, a complicated problem into a set of equations easy to solve by numerical method. Some assumptions to simplify the model are been done in order to present the process in few words; nevertheless, the technique here described can be extended with no difficulty to more realistic situations taking into account nonlinear spacers, absence of mechanical link between adjacent assemblies, shocks, etc. ... . The method may be also applied to heat exchangers and condensers.

\section{References}

[1.. BENSOUSSAN, A., LIONS, J.L., PAPANICOLAOU, G., "Asymptotic Analysis for Periodic Structures", Nortb-Holland, Ansterdam (1978).

[2] BlRGiAN D., LIONS, J.L., PAPANICOIAAOU, G., "Summer School on Homogenization", EDF-CEAINRIA, France (1983).

[3] CHEN, S.S., "Vibrations of Nuclear. Fue] Bundles", Nuc]. Eng. Design 35, 399-422 (1975).

[4] JAGANNATH, D.V., PAIDOUSSIS, M.P., "Solid-Fluid Interactions in the vibrations of Nuclear Fuel Bundle", Meeting on Vibrations in Nuclear Plant5, Keswick, U.K. (1978).

[5] PLANCHARD, J, IBNOU-ZAHIR, M., "Natural Frequencies of Tube Bundle in an Uncompressible Eluid", Comp. Math. Appl. Mech. Eng. 41, 47-68 (1983).

[6] PLANCHARD, J., "Modélisation du Comportement Dynamique des Assemblages Combustibles d'un Réacteur à Eau Pressurisée", E.D.F., Bulletin des Etudes et Recherches, Série C, 1, $31-41$ (1985).

[7] PLANCIIARD, J., "Développements Asymptotiques des Equations Dynamiques d'un Fajsceau Tubulaire Immergé", E.D.F., HI/4947-07 (September 1984).

[8_ CHEN, S.S., WAMBSGANSS, H.W., "Paralle1 - F]ow - Induced Vibrations of Fuel Rods", Nuc1. Eng. Design 18, 253-278 (1972).

[9]. PAIDOUSSIS, M.P., BESANCON, P., "Dynamics of Arrays with Internal and External Axial Flow", Journ. Sound. Vibrat. 76, 361-380 (1981).

[10_] SCHUMANN, U., BENNER, J., "Homogenized Model for Fluid-Structure Interaction in a Pressurized Water Reactor Core", Proceedings Sixth Intl. Conf. on Structural Mechanics in Reactor Technology, Paris, France, August 1981, Paper B5/7. 


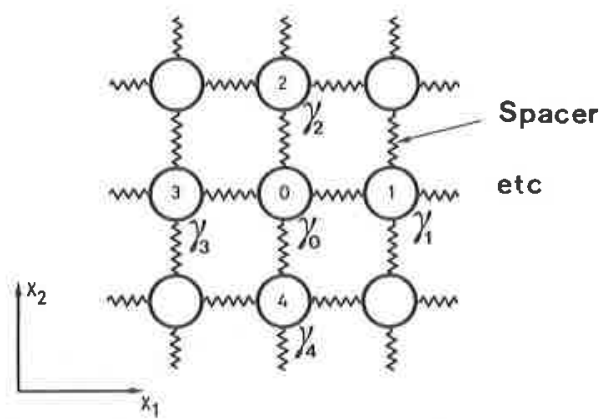

FIGURE 1 : Configuration of tubes and spacers.

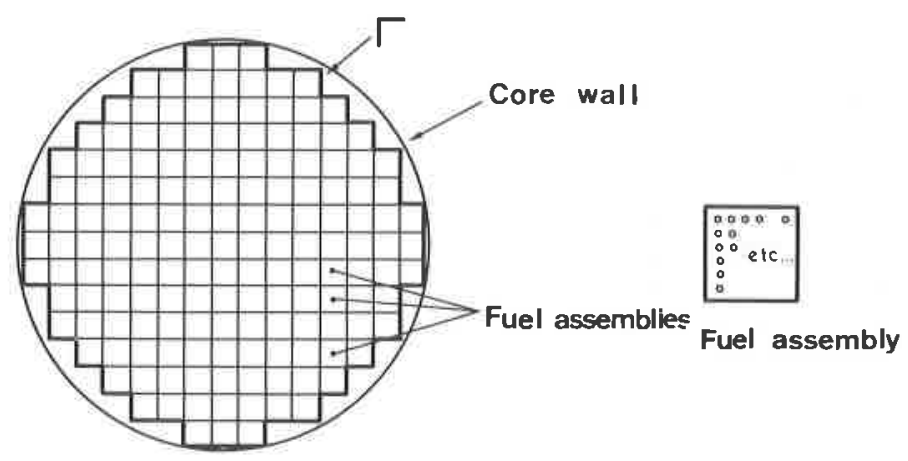

FIGURE 2 : Example of PWR core.

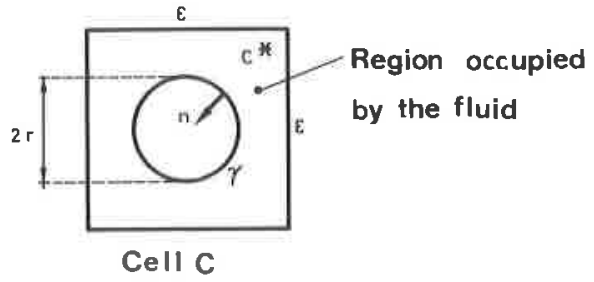

FIGURE 3 : Elementary cell for PWR core. 

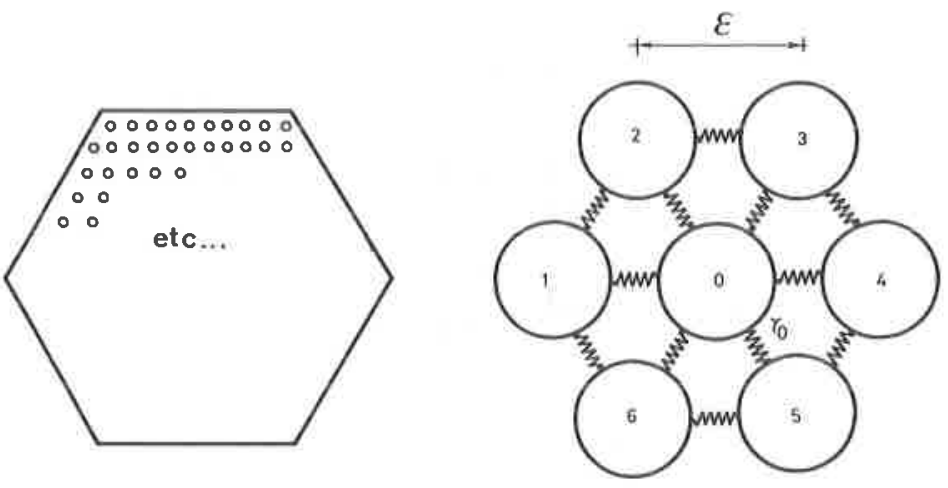

FIGURE 4 : Fuel assembly of fast breeder reactor.
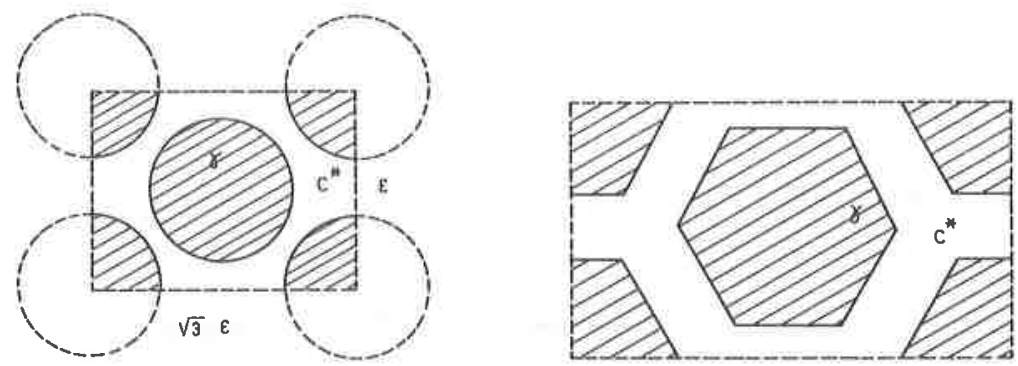

FIGURE 5 : Examples of elementary cells for fast breeder reactor (figures not to scale). 